\title{
AN ASPIRATION-LEVEL INTERACTIVE MODEL FOR MULTIPLE CRITERIA DECISION MAKING
}

\author{
Vahid Lotfi ${ }^{1} \dagger$, Theodor J. Stewart ${ }^{2} \ddagger$ and Stanley Zionts ${ }^{3} \S$ \\ ${ }^{1}$ School of Management, The University of Michigan-Flint, Flint, MI 48502, U.S.A., ${ }^{2}$ Department of \\ Mathematical Statistics, University of Cape Town, Cape Town, Republic of South Africa and ${ }^{3}$ School \\ of Management, State University of New York at Buffalo, Buffalo, NY 14260, U.S.A.
}

\begin{abstract}
Scope and Purpose - An approach is developed for solving decision problems involving the choice among discrete alternatives based on two or more conflicting objectives. The approach involves the user choosing levels of the objectives that he desires to achieve (levels of aspiration), and provides him with various kinds of feedback. For example, the user is told what fraction of alternatives satisfies his levels of aspiration. It also provides the user with rankings of alternatives based on one of two possible ranking schemes, and other kinds of feedback. According to the ranking schemes, the most highly ranked by the system will never be inferior to or dominated by another alternative. By this we mean that there will not exist another alternative that is at least as good as the most highly ranked in all respects, but strictly better in at least one respect. The theory for the proposed procedure has been drawn from work of various researchers.

The approach has been implemented in a computer program that is easy to use, and has been tested in a number of circumstances, including an experiment described in the paper. The results of the experiment are favorable to the method proposed.
\end{abstract}

\begin{abstract}
A simple, eclectic approach for solving discrete alternative multiple criteria decision problems is presented. It is based on the concept of the level of aspiration, and draws on ideas of various researchers. It assumes that the user has a set of alternatives with each alternative having a score on each of a number of objectives or measures of performance. The user determines his levels of aspiration for different objectives. $\mathrm{He}$ is then provided with considerable feedback as to the degree of feasibility of each level of aspiration as well as the degree of feasibility with respect to all levels of aspiration as a whole. The closest nondominated solution to the solution specified by the levels of aspiration is provided. The proposed method is easy to use and easy to understand and has been implemented on a personal computer (an IBM PC or compatible with $512 \mathrm{~K}$ RAM). We describe an experimental application in which 49 students in an MBA program used the method to solve two discrete alternative multiple criteria decision problems.
\end{abstract}

\section{INTRODISTION}

The management science literature is filled with models for solving multiple criteria decision making (MCDM) problems (see, for example, Hwang and Masud [1] and Evans [2]) decision problems involving more than one objective. Though there are applications of some of the methods in the literature, the methods are not as widely used as might be thought, particularly given the importance of the generic MCDM problem.

In this paper, we consider the specific problem of choosing one alternative from a set of alternatives. The decision maker has a set of attributes, criteria, or objectives that describe each alternative. Some of these are objective, and some are subjective. Each alternative has a measure of achievement for each objective. The motivation for this article was to have a simple model that can be related to and used by almost anyone.

Kepner and Tregoe [3] present a framework for analysis that is designed to help managers

$\uparrow$ Vahid Lotfi is Associate Professor of Management Science in the School of Management, The University of Michigan-Flint. $\mathrm{He}$ received his Ph.D. in Operations Research from SUNY at Buffalo. His work has appeared in Computers \& Operations Research, Decision Sciences, IIE Transactions, European Journal of Operational Research, and Journal of the Operational Research Society. Dr Lotfi is also a coauthor of Redundancy in Mathematical Programming and other textbooks on production and operations management and management science. His current research interests include multiple criteria decision making and applied management science.

¥Theodor Stewart is Professor of Statistical Sciences at the University of Cape Town, Republic of South Africa. He received the B.Sc. degree in Chemical Engineering from the University of Cape Town, and M.Sc. and Ph.D. degrees from the University of South Africa in Operations Research/Statistics. From 1976 to 1984 he was Head of Operations Research and Statistics at the South African Council for Scientific and Industrial Research, and has been President of both the Statistical Association and the Operations Research Society of South Africa. His research interests are in MCDM and decision theory, particularly with application to natural resources management, and he has published in Management Science, Naval Research Logistics, Journal of the Operational Research Society, European Journal of Operational Research, and IEEE Transactions on Systems, Man and Cybernetics.

$\S$ Stanley Zionts. Professor Zionts' biographical information appears elsewhere in this issue. 
systematically make decisions. It is essentially a checklist that helps the user determine the important aspects of a decision, and structure the payoffs and probabilities. The authors were successful in offering executive training programs built around their approach. They classify objectives as musts or most important; wants or desirable, but capablc of being traded off; and ignores or may bc ignored. Newsted and Wynne [4] implemented the approach of Kepner and Tregoe [3] in a BASIC computer program that is an early application of artificial intelligence.

Our approach is modest. Though we use some of the Kepner and Tregoe [3] ideas (independently developed), we use them somewhat differently. We assume a deterministic problem, and use various ideas to come up with a simple way of choosing one alternative from a set of alternatives. Most people can relate to the multiple criteria decision making problem we have chosen. Indeed, choosing a university in which to enroll as a student, choosing a car to buy, and choosing a house to buy are all examples of such problems.

Many of the methods of multiple criteria decision making can be seen as normative models in that they prescribe ways in which decision makers should make decisions (for example, as if they used a utility function). We can think of normative models as one extreme, whereas the other extreme is descriptive models, or models of how people make decisions. When most people go shopping for groceries, for example, they use lists. Once a consumer takes his list to the market, he may suppress the influence of price, and other factors, or he may consider them only in a secondary manner. The task at that point is to simply buy everything on the list (however, some items on the list may be listed by brand, size, etc.).

We call our approach the aspiration-level interactive method (AIM). It is neither normative nor descriptive. It is a decision tool designed to assist a user in exploring the decision alternatives in a user-driven manner, generating nondominated $\dagger$ solutions that are close (in some sense) to the user's levels of aspiration. AIM is eclectic in nature; it draws from the results of many researchers. It includes concepts based on goal programming, tradeoffs, and other aspects of MCDM. Our objectives in developing AIM are to have a procedure that is dynamic and interactive, that can be easily used by anyone.

AIM is designed to solve the deterministic decision analysis problem (see Chankong et al. [5]) though it can be extended to deterministic mathematical programming problems. It assumes a matrix ( $n$ by $p$ ) of alternatives and objectives where each row constitutes an alternative and each column constitutes an objective. We assume the user wants to choose precisely one alternative. The objectives may be cardinal or ordinal, and the entry in row $i$ and column $j$ of the matrix is the measure of performance on objective $j$ of alternative $i$. AIM does not require any assumption about the nature of the objectives and can solve problems with three types of objectives as follows:

(1) those that are to be maximized

(2) those that are to be minimized

(3) those for which deviations in either direction from some target value, or range of values (see below) are to be minimized (e.g. an individual wants to find a house that is not too far from shopping, but not too close).

For each of the three objective function types, and especially the third, there may be associated satisficing thresholds, such that the decision maker is effectively indifferent to values above or below the threshold (in the case of the first two objective types) or within the range of threshold values in the third case (in the example of the proximity of shopping example given above).

We use levels of aspiration to explore the nondominated solution frontier by allowing the user to establish levels of aspiration and then know something about the reasonableness of such aspirations. This idea comes from Herbert Simon's ideas of bounded rationality and satisficing (March and Simon [6]). More specifically, AIM provides a measure of the fraction of all solutions that satisfies those levels, considering the objectives one at a time and together. It is enhanced by suggestions of other "nearby" solutions, information about the degree of attainment of various alternative levels, and related information that we believe to be useful to people making decisions.

What is lacking in our procedure is some of the more formal aspects of multiple criteria decision

† One solution dominates another if the first solution is at least as good as the second in every criterion and strictly better in at least one of them. Solutions that are not dominated by any other solutions are called nondominated solutions. 
making. What can we say about the optimality of a decision? What can we say about convergence? We can say roughly what Wierzbicki [7] says: we can identify nondominated solutions, and help the user choose from the solutions. It is not important that the user choose such a solution, as we described above; it is sufficient that he be aware of the existence of such solutions and the tradeoffs among them.

The paper consists of four sections. In this section, we have overviewed our approach. In Section 2 , we develop the methodology of the approach. In the third section we describe a comparative study of the method. The final section summarizes the paper.

\section{METHODOLODY}

As mentioned, our approach is based on utilizing levels of aspiration. The user adjustes the level of aspiration for each objective while obtaining feedback on their "reasonableness". AIM then provides the "closest" nondominated solution to the aspiration levels as suggested by Wierzbicki [7]. To further explore the set of nondominated solutions, we identify nearby solutions to the closest nondominated solution through a simplified variation of the outranking procedure used in the Electre methods (see Roy [8]).

\subsection{Problem definition}

We have defined the MCDM problem in terms of objectives (of three possible types, with or without satisficing thresholds), alternatives and evaluations (cardinal or ordinal scales) of each alternative in terms of each objective. For each objective, we assume a must level, or a constrained level that must be achieved, no matter what, a want, desired, or aspiration level, that is desired but capable of compromise, and an ignore level (or threshold), beyond which satisfaction is not further increased. Any ordinal objectives are given values to represent their levels. Except for their order, the values assigned to respresent each level are not important. For ease of notation in describing the methodology only (though not in the method), we shall assume that objectives with a target value:

(i) are represented as two objectives, a maximizing one (to achieve the target level or range from below); and a minimizing one (to achieve the target level or range from above);

(ii) always have thresholds explicitly stated, with the lower end of the range (the threshold for the maximizing part of the objective) not exceeding the upper end of the range; equality of these thresholds implies a precise target (the number of bedrooms desired in a house, for example), and inequality a target range (the proximity of shopping, for example).

Every objective therefore is assumed to be either a maximizing or minimizing objective (having split any target objectives into two sub-objectives though we do not do this in the method). More formally, let:

$T_{i}=$ represent the satisficing threshold for objective $i$

$z_{i}^{k}=$ be the value of alternative $k$ in terms of objective $i$

$A_{i}=$ be an aspiration level (or desired level of performance) for objective $i$, which should not, in order to be meaningful, exceed the ideal value (see below) for a maximizing objective, and vice versa.

Further, we define the ideal $\left(I_{i}\right)$ and nadir $\left(N_{i}\right)$ values for objective $i$ as:

$$
\begin{aligned}
I_{i} & =\left\{\begin{array}{l}
\min \left[T_{i}, \max _{k}\left\{z_{i}^{k}\right\}\right] \text { if } i \text { is maximizing } \\
\max \left[T_{i}, \min _{k}\left\{z_{i}^{k}\right\}\right] \text { if } i \text { is minimizing }
\end{array}\right. \\
N_{i} & =\left\{\begin{array}{l}
\min \left\{z_{i}^{k}\right\} \text { if } i \text { is maximizing } \\
\max \left\{z_{i}^{k}\right\} \text { if } i \text { is minimizing }
\end{array}\right.
\end{aligned}
$$

For the maximizing objective $i$, the ideal and nadir values provide upper and lower bounds on $A_{i}$. The bounds should be reversed for the minimizing case. $I_{i}$ and $N_{i}$ are used to provide DM with a range of acceptable values for $A_{i}$. 


\subsection{The solution approach}

The $z_{i}^{k}$ values are ordered from the least to most preferred, for each objective $i$. This allows us to easily extract such quantities as the median and quartile values for each objective.

The decision maker is provided with the following set of "basic information":

(a) $A_{i}$, the current goal (or aspiration level) for objective $i$, initially set to the median, together with the proportion of alternatives that are at least as good as this value

(b) two other aspiration levels; (1) the next better level than $A_{i}$, and (2) the next worse level than $A_{i}$. (Each is the next respective different value occurring in the data base.)

(c) the ideal (best possible) and nadir (worst possible) values for each objective [equations (1) and (2)]. The ideal solution is the name given to a (fictitious) $\dagger$ solution that achieves all best values, and the nadir solution is the name given to a fictitious solution that achieves all worst valucs

(d) the proportion of alternatives that simultaneously satisfy the given aspiration levels $\lceil(\mathrm{a})$ and $(\mathrm{b})\rceil$

(e) a "nearest nondominated solution", defined to be the best alternative according to a scalarizing function proposed by Wierzbicki [7], with a weight on criterion $i$ given by $\left(A_{i}-N_{i}\right) /\left(I_{i}-N_{i}\right)$ (valid for both maximization and minimization objectives) using a Tchebycheff function. The weights are set to reflect the increasing importance attached to objective $i$ as the aspiration level is moved closer to the ideal (see Appendix A for details of the algorithm).

Other metrics may be used instead of the Tchebycheff function used in (e). A linear metric is not recommended because it cannot yield a nondominated but convex dominated solution $\ddagger$ as the "most preferred".

A number of options are available to the user. First, the user can change his current goal levels, and obtain an updated set of basic information. The levels for each objective may only be set to realizable vaues (values that occur in the data base). As the aspiration levels are changed, the nearest solution changes. Second, the user can scan the solutions satisfying his aspiration levels. He can rank all alternatives at any time, according to the closeness to the current goal levels using the scalarizing function of step (e). The user can also request a set of "neighboring" solutions. We use a simplified version of outranking as used in the electre methods to find "neighbors" of the nearest solution. Alternative $j$ is said to outrank alternative $k$, if and only if the following conditions are satisfied:

(i) the fraction of objectives, for which $j$ is at least as good (any objective value in the indifference region is considered as having the ideal value, and hence is as good as any other) as $k$, is at least $1 / 2$

$$
\operatorname{Max}_{i}\left\{\left(\mathrm{z}_{i}^{k}-z_{i}^{j}\right) /\left(I_{i}-N_{i}\right)\right\} \leqslant c
$$

where the user chooses $c$ implicitly by adjusting the number of "neighbor" solutions desired (see Appendix B for details of the procedure).

In other words, alternative $j$ outranks alternative $k$ if $j$ has better scores on at least half the objectives and for those objectives which $k$ has better scores, the normalized difference (between scores of alternative $k$ and alternative $j$ ) is not greater than $c$ (in our implementation $c$ is initially set at $20 \%$ but the user changes its value). Intuitively, one solution is said to outrank another if the first is at least as good as the second in most respects, and not too much worse in any one respect. We have chosen this simplification because it captures much of the spirit of outraking, without forcing the user to appreciate the meaning of the parameters involved. We have the option of classifying the alternatives as dominated and nondominated, and if desired, deleting the dominated alternatives. Finally, we have the option of exploring the distribution of each of the problem objectives. This may be done in terms of cumulative distributions of the objectives, or in terms of quartiles. 


\section{EXPERIENCE WITH THE METHOD}

The performance of AIM was evaluated in a laboratory experiment using student decision makers. The purpose of the experiment was to compare three approaches:

(1) a computer implementation of AIM

(2) Expert Choice, a computer implementation of the analytic hierarchy procedure (Saaty [9])

(3) a manual procedure that has the user choose an alternative from a list giving all alternatives and their attributes.

We wanted to compare the procedures, their ease of use, the quality of the solution produced, and other measures of effectiveness. Below we present a summary of the results of the experiment.

The experiment consisted of having students use all three approaches to solve two discrete alternative MCDM problems. The subjects were 49 second-year MBA students at the State University of New York at Buffalo: 20 women and 29 men. They were all familiar with the use of microcomputers, and had taken courses in quantitative methods and information systems. None of the subjects (see below) had indicated prior experience with either AIM or Expert Choice (EC). The identities of the developers of the systems were scrupulously withheld from the subjects. Because of irregularitics, two subjects had their results climinated from our analyses. One student did have prior experience with Expert Choice. Even though his results were more favorable towards AIM, we excluded them. Another subject completed the AIM section but encountered errors with EC and did not complete the experiment. His results were also excluded. In what follows, the analysis of the results are based on responses from the remaining 47 subjects.

\subsection{Description of the decision problems}

Two decision problems were utilized. The first problem (I) was taken from Zeleny [10]. It involved evaluating 33 washing machines on the basis of four criteria: price, total wash time, power consumption, and water consumption. All of the four objectives were to be minimized. None of the alternatives (machines) were dominated.

The second decision problem (II) consisted of selecting a personal computer for purchase among 124 personal computers. The data for the personal computers were collected from several popular personal computer magazines. The PCs were evaluated on five criteria: price, quantity of random access memory, disk storage, processor speed, and type of display. The first objective, price, was to be minimized, and the remaining four objectives were to be maximized. Of the 124 alternatives, 61 were dominated. The structure of the two problems for AHP is shown in Figs 1 and 2.

\subsection{Experimental setting}

The subjects, all students in a graduate course on Management Information Systems, were introduced to the topic of decision support systems via readings and lectures. They were then

Selecting a Washing Machine

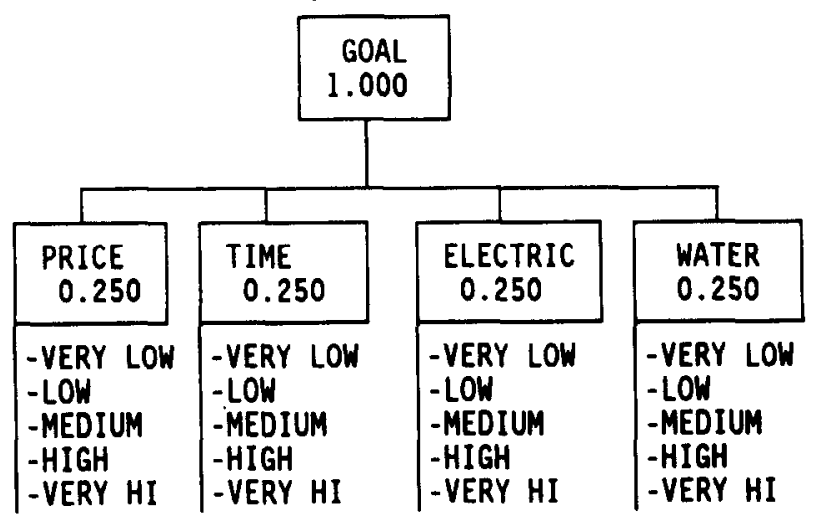

Fig. 1. The AHP structure for the washer problem. 


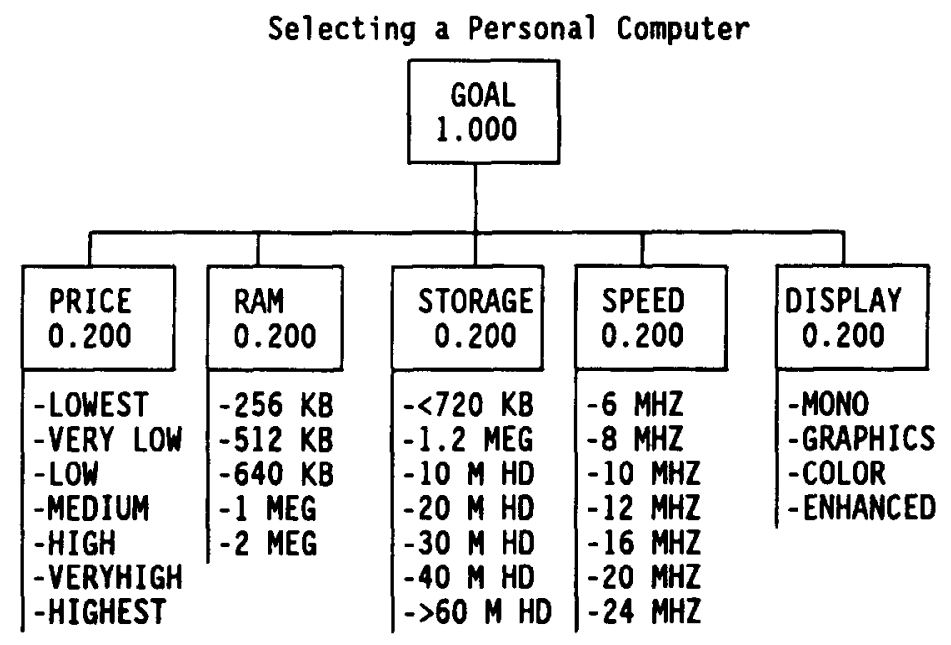

Fig. 2. The AHP for the $\mathrm{PC}$ problem.

presented with a lecture in which both the reading assignments on MCDM methods as well as discrete alternative MCDM methods were discussed. During a second lecture, they were given a tutorial demonstration of both AIM and EC. Each presentation was presented in 40 min, and every effort was made to present the two approaches in the same light. For the tutorial, the decision problem involved the purchase of a house from a set of 31 houses. The houses were evaluated on four criteria: number of bedrooms, number of bathrooms, age, and list price. The first two objectives were to be maximized and the last two objectives were to be minimized. Computer programs were used for each.

At the end of the tutorial, an instruction package, containing a task description, a work sheet, two questionnaires, and printed lists of alternatives for both problems I and II was given. In the task description, it was stated that there were no right or wrong answers and that the purpose of the study was to compare the MCDM methods and their implementations involving a choice among alternatives. The task description also contained descriptions of the two decision problems. Subjects were asked to use the two methods to solve the two problems in the order assigned to them in class. An orthogonal design was utilized whereby the order of the methods as well as the problems were varied.

Expert Choice can produce inconsistent weight structures (see Saaty [9]). During the tutorial session, the participants were shown how to use EC to resolve inconsistencies. In the instruction sheet, they were asked to resolve any inconsistencies above a recommended 0.1 threshold.

The work sheet contained entires for recording the start times, stop times, and most preferred choices, for each method and each problem. The participants were also given an opportunity to select most preferred solutions for each problem, independent of the earlier part of the experiment. They were instructed to have available the printed lists of alternatives with their performance levels indicated for use in this phase. They then entered their most preferred choices. The choices could be the same as those suggested by either method or they could be chosen by scanning the list of alternatives. In what follows, we will refer to the most preferred solution chosen in this final phase as the final choice (FC).

\subsection{The questionnaire}

The subjects were asked to fill out a questionnaire, designed to evaluate the methods, after completing the experiment. Twenty questions were used to evaluate the performance of each method on a multi-dimensional scale. The questions, designed to measure user satisfaction, were extracted from Doll and Torkzadeh [11] and Baroudi and Orlikowski [12]. They comprised five dimensions including content, accuracy, format, ease of use, and timeliness. As suggested by the authors, the ratings for the twenty questions consisted of a five-point Likert scale. 


\subsection{Analysis of the results}

We hypothesized that AIM would do better than both Expert Choice and the manual approach. The null hypothesis was that AIM and each of the other two methods were identical in performance, and the alternate hypothesis was that AIM was superior to each. We used three measures of performance:

(1) the quality of the solution obtained

(2) the agreement of choice among methods

(3) the preference of the participants for the methods.

We also hypothesized that between AIM and Expert Choice, AIM would outperform Expert Choice as a predictor of the final choice.

3.4.1. Quality of solutions. There are several ways of measuring the quality of the solutions obtained. First, we measured the percentage of nondominated solutions obtained by each method. Problem I (the washing machine problem) did not include any dominated solutions. For problem II (the PC problem), the breakdown of participants who chose nondominated solutions consisted of: $98 \%$ (46 participants) using AIM, $91 \%$ (43 participants) using EC, and $79 \%$ (37 participants) for the FC. Statistically, the number of dominated solutions obtained by EC was not significantly less (at the 0.01 level) than that of FC. However, the number of dominated solutions obtained by using AIM was significantly less (at the 0.01 level) than the number obtained by FC. The one participant who selected a dominated solution using AIM chose an IBM computer. Presumably, the choice was made because of the IBM name. Of the four participants who chose dominated solutions using EC, half chose IBM computers. The other two chose computers whose names were not well known. (In the database, all names but IBM and Epson were sufficiently changed as to be unidentifiable by the users. This was not done deliberately, but occurred because of the truncation of the names by which the computers were coded in the database. In the experiment, no one chose a dominated Epson computer.)

Because of its additive structure, it is possible for EC to be unable to choose a nondominated but convex dominated solution as most preferred, whereas AIM can. A simple example of this is a two-objective, three-alternative problem in which both objectives are to be maximized. Using a range for each objective as zero to ten, suppose that the alternatives have values $(10,0)\left(\varepsilon_{1}, \varepsilon_{2}\right)$, and $(0,10)$, respectively, where $\varepsilon_{1}>0$ and $\varepsilon_{2}>0$ are sufficiently small compared to 10 so that the decision maker's marginal utility of 10 is greater than two times that of either $\varepsilon$. Then, no additive function can prefer the second alternative to either the first or third. (The second alternative is convex dominated through nondominated.) EC can only rank the first or third alternative as the most preferred, whereas AIM can rank any of the three as most preferred.

Another difficulty associated with the use of AHP is that the ranking of the alternatives, in certain situations, can be arbitrary (see Dyer [13]). The occurrence of this phenomenon in AHP, referred to as "rank reversal", was first observed by Belton and Gear [14]. They suggest that for a pair of criteria $C_{i}$ and $C_{j}$, if $C_{i}$ is preferred to $C_{j}$ (as determined by AHP) when considering alternative $k$, the preference is not guaranteed to remain the same and can be reversed by inclusion of another alternative. Dyer [13] proposes an approach based on the traditional methods of analysis (e.g. MAUT) for alleviating the occurrence of rank reversal in AHP (see Saaty [15], Dyer [13], and Harker and Vargas [16] for a recent debate on the validity of the alternative ranking through the use of AHP).

Table 1 shows the ratio of the method standard deviation to the population standard deviation for each method and for each objective for each of the two problems. The smaller this measure, the less variation there is in the results found by the user. If most of the participants had had only small deviations from the initially provided conditions of each of the methods, then these figures would be small. Apparently, the participants were actively involved in the choice of alternatives in the methods, because their standard deviations relative to the population were high. Further, the students found that they could better relate to problem II than to problem I. Most of the students had considered the purchase of a personal computer, whereas few had considered the purchase of a washing machine. After we had performed the experiment, we learned that problem I had earlier been criticized as an experimental problem (see Korhonen et al. [17]). 
Table 1. Ratio of method standard deviation to population standard deviation

\begin{tabular}{clccc}
\hline Problem & Objective & AIM & EC & FC \\
\hline I & Price & 0.82 & 0.49 & 0.91 \\
& Time & 0.85 & 0.96 & 1.01 \\
& Electric & 0.87 & 1.03 & 1.03 \\
& Water & 0.50 & 0.97 & 0.68 \\
\hline II & Price & 1.22 & 2.41 & 1.08 \\
& RAM & 0.97 & 1.61 & 0.96 \\
& Sturage & 1.40 & 2.09 & 0.72 \\
& Speed & 1.02 & 1.41 & 1.05 \\
& Display & 0.75 & 1.11 & 0.80 \\
\hline
\end{tabular}

Table 2. Consistency of method and final choice (FC)

\begin{tabular}{lcc}
\hline & \multicolumn{2}{c}{ Problem } \\
\cline { 2 - 3 } Method & I & II \\
\hline AIM $=$ FC & 6 & 3 \\
$\mathrm{EC}=\mathrm{FC}$ & $(13 \%)$ & $(6 \%)$ \\
$\mathrm{AIM}=\mathrm{FC}$ & 20 & 24 \\
$\mathrm{EC} \neq \mathrm{FC}$ & $(43 \%)$ & $(51 \%)$ \\
$\mathrm{AIM} \neq \mathrm{FC}$ & 9 & 3 \\
$\mathrm{EC}=\mathrm{FC}$ & $(19 \%)$ & $(6 \%)$ \\
$\mathrm{AIM} \neq \mathrm{FC}$ & 0 & 1 \\
$\mathrm{EC} \neq \mathrm{FC}$ & $(0 \%)$ & $(2 \%)$ \\
$\mathrm{AIM}=\mathrm{EC}$ & & 16 \\
AIM $\neq \mathrm{FC}$ & 12 & $(34 \%)$ \\
$\mathrm{EC} \neq \mathrm{FC}$ & & \\
AIM $\neq \mathrm{EC}$ & $(26 \%)$ & \\
\hline $\begin{array}{l}\text { The number and percentage give the number and percentage } \\
\text { of users whose final choice corresponded to the solution }\end{array}$ \\
found by the indicated method and problem. I Ihe \\
percentages of each problem do not add to $100 \%$ \\
respectively because of rounding.
\end{tabular}

3.4.2. Agreement of choices using the methods with subjects' FC. We compared the agreement between the choice by subject of solutions using each method and their final choices (FC). Table 2 presents the number and percentage of participants in each category. The observations in each row are mutually exclusive and collectively exhaustive. They relate the number of solutions satisfying the given characteristics. The first row gives, for example, the number of participants and the percentage for which the user found the same solution using all three methods. Using the first and second rows, we can see that the AIM solution agreed with the final choice for 26 and 27 participants for problems I and II, respectively. Using the first and third rows, we see that the EC solution agreed with the final choice for 15 and 6 participants, respectively. (The total percentages for each problem do not add to $100 \%$ because of round off error.) There was no significant difference at the 0.01 level between solutions obtained using AIM and FC (for both problems). The results were the same for EC for problem I. However, for problem II, the EC results were different (at the 0.05 level) from FC. One possible explanation for this is that the participants were more interested in the outcome of problem II as mentioned above (this is further validated by noting the amount of time spent on each problem as discussed below). Consequently, they spent more effort in finding a "most preferred" solution. As seen from the table, a substantial portion of the participants (about $36 \%$ ) selected a PC different from those chosen using either AIM or EC. However, of those who selected a PC that was the same as that chosen using either AIM or EC, most ( 27 out of 30) chose a PC suggested by AIM. Only 6 of these participants selected a PC suggested by EC.

A more comprehensive test was conducted to compare the solutions chosen by AIM and FC, and EC and FC (see Table 3). We used a test of comparison of several multivariate means (see Johnson and Wichern [18]). For AIM vs the final choice (FC), we could not reject the null hypothesis that the two solutions were the same at the 0.01 level, whereas for EC vs the final choice (FC), we did reject the null hypothesis that the two solutions were the same at the 0.01 level.

As mentioned, the subjects were asked to record the start time and stop time for each problem and each method. Table 4 presents the mean, standard deviation, minimum, and maximum amount of time spent on each problem by each method. The average amount of time spent on each problem by each method is about $15 \mathrm{~min}$ except for problem II using EC. In this case, the average time is about $21 \mathrm{~min}$. There was no significant difference between time spent on problem I and problem II using AIM. For EC, time spent on solving problem II was significantly more at the 0.01 level than the time spent on problem I. The reason is that the subjects were more interested in the outcome of problem II and hence needed more time to obtain a truly preferred solution when using EC.

3.4.3. Attitudes towards the methods. Two types of statistical analysis were performed on the results of the questionnaires. First, a common factor model was applied to the results of both AIM 
Table 3. Difference between solutions from FC and $\mathrm{AIM}$ and $\mathrm{FC}$ and $\mathrm{EC}$ (problem II)

\begin{tabular}{lcc}
\hline Objective & FC - AIM & FC - EC \\
\hline Price & 116.3 & -1444.4 \\
& $(1186)$ & $(2831)$ \\
RAM & 22.5 & -404.4 \\
& $(265)$ & $(571)$ \\
Storage & -2.5 & -39.8 \\
& $(39)$ & $(73)$ \\
Speed & -0.2 & -1.2 \\
& $(3)$ & $(6)$ \\
Display & -0.3 & 0.3 \\
& $(1)$ & $(2)$ \\
\hline
\end{tabular}

Table entries represent mean and standard deviations for the differences between characteristics of the solutions proposed by each method and final choice.
Table 4. Time spent on each problem by each method $\dagger$

\begin{tabular}{llrr}
\hline \multirow{3}{*}{ Method } & & \multicolumn{2}{c}{ Problem } \\
\cline { 3 - 4 } & & \multicolumn{1}{c}{ I } & \multicolumn{1}{c}{ II } \\
\hline \multirow{4}{*}{ AIM } & Mean & 16.1 & 15.9 \\
& SD & 10.7 & 10.1 \\
& Minimum & 3.0 & 2.0 \\
& Maximum & 45.0 & 40.0 \\
\hline \multirow{3}{*}{ EC } & Mean & 16.5 & 21.2 \\
& SD & 9.2 & 15.9 \\
& Minimum & 5.0 & 5.0 \\
& Maximum & 37.0 & 82.0 \\
\hline
\end{tabular}

† Times are in minutes.

and EC to determine the dimentionality of the responses. The method of maximum likelihood estimators was utilized. There were seven significant factors (at the 0.05 level) with the seventh factor having no significant loading. Consequently, a second factor analysis was performed with the number of factors fixed at six. The six common factors collectively explained about $75 \%$ of the variance. The six common factors were then rotated, using the "varimax" criterion (see Dillon and Goldstein [19]). The first factor comprised the general effect explaining about $38 \%$ of the total variance (over $50 \%$ of the variance explained by the common factors). The general effect common factor included characteristics relating to: precision of information; making a better decision; and improving quality of decision. The second common factor related to the user friendliness of the computer program. The third factor represented quality of information produced. The fourth factor related to the quality of output (program interface). The fifth common factor represented the overall satisfaction, and sixth represented sufficiency of information produced. The factor model indicated that the questionnaire did in fact form a multi-dimensional scale as suggested by Doll and Torkzadeh [11].

The second statistical analysis consisted of comparing the responses for the AIM survey with those of the EC. A paired $t$-test was utilized to determine the statistical differences between the mean responses. The results indicated that there was no significant difference at the 0.01 level between AIM and EC with respect to four characteristics. These consisted of clarity of information, user friendliness, helping the DM to set his priorities, and speed of the program. The remaining sixteen responses were significantly in favor of AIM at the 0.01 level. "Quality of the solution" was the most preferred characteristics of the AIM compared to EC. This is in agreement with the earlier finding that more participants chose the AIM solutions as their final choices than those choosing EC solutions. Three other features of AIM were also strongly preferred (at the 0.0001 level) to EC. They included sufficiency of information, accuracy of information, which enabled users to make better decisions.

\section{SUMMARY}

We have developed a simple, eclectic approach for solving discrete alternative multiple criteria decision problems. It is based on the concept of levels of aspiration, and draws on ideas of various researchers. The user determines his levels of aspiration for different objectives in an interactive personal computer environment in which he is given considerable feedback as to the degree of feasibility of each level of aspiration as well as the degree of feasibility with respect to all levels of aspiration as a whole. The closest nondominated solution to the solution specified by the levels of aspiration is provided, as are other useful outputs. We have developed a method based on these ideas that is easy to use and easy to understand, and have implemented the approach on a personal computer (an IBM PC or compatible with 512K RAM). An application of the method comparing AIM with EC showed that the approach is about the same with respect to clarity of information, 
user friendliness, setting priorities, and speed of operation. On numerous measures, AIM outperformed EC, but for no measures was the reverse true.

Acknowledgement - The authors wish to thank the anonymous reviewer for his/her helpful suggestions.

\section{REFERENCES}

1. C. L. Hwang and A. S. M. Masud, Multiple Objective Decision Making-Methods and Applications, a State-of-the-Art Survey. Springer, Berlin (1981).

2. G. W. Evans, An overview of techniques for solving multiobjective mathematical programs. Mgmt Sci. 30, 1268-1282 (1984).

3. C. H. Kepner and B. B. Tregoe, The Rational Manager. McGraw-Hill, New York (1965).

4. P. R. Newsted and B. E. Wynne, Augmenting man's judgment with interactive computer systems. Inst. J. Man-Mach. Stud. 8, 29-59 (1976).

5. V. Chankong, Y. Y. Haines, J. Thadathil and S. Zionts, Multiple criteria optimization: a state of the art review. In Decision Making with Multiple Objectives (Edited by Y. Y. Haimes and V. Chankong), pp. 39-90. Proceedings, Cleveland, Ohio, 1984, 242 in Lecture Notes in Economics and Mathematical Systems, Springer, Berlin (1985).

6. J. March and H. A. Simon, Organizations. Wiley, New York (1958).

7. A. P. Wierzbicki, The use of reference objectives in multiobjective optimization. Working Paper 79-66, International Institute for Applied Systems Analysis, Laxenburg, Austria (1979).

8. B. Roy, Classement et choix en presence de points de vue multiples (la methode ELECTRE). Rev. Informat. Rech. Oplle 8, 57-75 (1968).

9. T. L. Saaty, The Analytic Hierarchy Process. McGraw-Hill, New York (1980).

10. M. Zeleny, Multiple Criteria Decision Making, pp. 210-211. McGraw-Hill, New York (1982).

11. W. J. Doll and G. Torkzadeh, The measurement of end-user computing satisfaction. MIS Q. 12, 259-274 (1988).

12. J. J. Baroudi and W. J. Orlikowski, A short-form measure of user information satisfaction. JMIS 4, 45-59 (1988).

13. J. S. Dyer, Remarks on the analytic hierarchy process. Mgmt Sci. 36, 249-258 (1990).

14. V. Belton and T. Gear, On a short-coming of Saaty's method of analytic hierarchies. Omega 11, 228-230 (1983).

15. T. L. Saaty, An extension of the AHP in reply to the paper 'Remarks on the analytic hierarchy process'. Mgmt Sci. 36, $259-268$ (1990).

16. P. T. Harker and L. G. Vargas, Reply to 'Remarks on the analytic hierarchy process' by J. S. Dyer. Mgmt Sci. 36, 269-273 (1990).

17. P. Korhonen, H. Moskowitz and J. Wallenius, Choice behavior in interactive multiple criteria decision-making. Working Paper, June (1987).

18. R. A. Johnson and D. W. Wichern, Applied Multivariate Statistical Analysis. Prentice-Hall, Englewood Cliffs, NJ (1988).

19. W. Dillon and M. Goldstein, Multivariate Analysis: Methods and Applications. Wiley, New York (1984).

\section{APPENDIX A}

\section{Algorithm Nearest}

The following algorithm finds the nearest nondominated solution for a given aspiration level. The initial starting solution is the nearest point to the median value of each objective. The algorithm terminates when the aspirations in two consecutive iterations are the same.

Step 0. Pre-processing.

(A) Determine the ideal value $\left(I_{i}\right)$ for each objective [equation (1)]

(B) Determine the nadir value $\left(N_{i}\right)$ for each objective [equation (2)].

We assume $I_{i} \neq N_{i} \forall i$, otherwise $j$ with $I_{j}=N_{j}$ is a "must" objective. Otherwise, all alternatives with $z_{j}^{k} \neq I_{j}$ are removed from further consideration, and the ideal and nadir values are updated. (C) Define:

$$
\begin{aligned}
& z_{i}^{\prime k} \begin{cases}z_{i}^{k} & \text { if } z_{i}^{k}<I_{i} \text { and } i \text { is maximizing } \\
I_{i} & \text { if } z_{i}^{k} \geqslant I_{i} \text { and } i \text { is maximizing }\end{cases} \\
& z_{i}^{r k} \begin{cases}z_{i}^{k} & \text { if } z_{i}^{k}>I_{i} \text { and } i \text { is minimizing } \\
I_{i} & \text { if } z_{i}^{k} \leqslant I_{i} \text { and } i \text { is minimizing. }\end{cases}
\end{aligned}
$$

(D) Let $z_{i}^{[k]}$ be a ordered set of $z_{i}^{\prime k}$ such that $z_{i}^{[1]}<z_{i}^{[2]}<\cdots<z_{i}^{[m i]}$,

where $m_{i}$ is the number of distinct values for objective $i$ between $N_{i}$ and $I_{i} ; m_{i}>1$ since $I_{i} \neq N_{i}$. Let $j=0$, and

$$
A_{i}^{j}= \begin{cases}z_{i}^{[\lfloor(m i+1) / 2\rfloor]} & \text { if } i \text { is maximizing } \\ z_{i}^{T \Gamma(m i+1) / 2\rceil]} & \text { if } i \text { is minimizing }\end{cases}
$$

continue with the Step 1 . 
Step 1. Main step.

(E) Find the set of nearest solutions: $Q_{j}=\left\{r \mid q^{r} \leqslant q^{k} \forall k\right\}$,

where

$$
\begin{aligned}
q^{k} & =\max _{i}\left\{w_{i}^{j} d_{i}^{j}+\varepsilon \Sigma_{r} w_{r}^{j} d_{r}^{j}\right\} \\
w_{i}^{j} & =\left(A_{i}^{j}-N_{i}\right) /\left(I_{i}-N_{i}\right) \\
d_{i}^{j} & =\left(A_{i}^{j}-z_{i}^{k}\right) /\left(I_{i}-N_{i}\right) .
\end{aligned}
$$

(F) Present $Q^{j}$ to DM. Let $j \leftarrow j+1$, and request $A_{i}^{j}$. If $A_{i}^{j}=A_{i}^{j-1} \forall i$, stop. Otherwise, repeat Step 1.

\section{APPENDIX B}

\section{Heuristic Outrank}

The following heuristic finds a set of outranking solutions for a given current solution. The cardinality of the set is controlled by the decision maker. The heuristic stops either when there are no outranking solutions, or the number of outranking solutions is within a range provided by the decision maker.

Let $\left[\mathrm{O}_{1}, \mathrm{O}_{2}\right]$ be the desired range for the number of outranking solutions (provided by the decision maker), define the binary function $\alpha_{j}^{k, c}$, for a pair of alternatives $c$ and $k$, as follows:

$$
x_{j}^{k, c}= \begin{cases}1 & \text { if } z_{j}^{\prime k} \geqslant z_{j}^{\prime c} \text { and } j \text { is maximizing, or } \\ & \text { if } z_{j}^{\prime k} \leqslant z_{j}^{\prime c} \text { and } j \text { is minimizing, } \\ 0 & \text { otherwise. }\end{cases}
$$

Further, let

$$
\beta_{j}^{k, c}= \begin{cases}\left(z_{j}^{\prime c}-z_{j}^{\prime k}\right) /\left(I_{j}-N_{j}\right) & \text { if } \alpha_{j}^{k, c}=0 \\ -\infty & \text { otherwise. }\end{cases}
$$

The steps of the heuristic are as follows:

Step 0.0. Let $\Gamma \leftarrow \varnothing, k \leftarrow 1, s \leftarrow 0$, and $t \leftarrow 1$.

Step 0.1. If $\left.\Sigma_{j} \alpha_{j}^{k, c} \geqslant \Gamma(p+1) / 2\right\rceil$, let $\Gamma \leftarrow \Gamma \cup\{k\}$.

Step 0.2. Let $k \leftarrow k+1$. If $k \leqslant n$, go to Step 0.1 .

Step 1.0. If $\Gamma=\varnothing$, stop. There are no outranking solutions to alternative $c$. Otherwise, let $\Gamma^{\prime} \leftarrow \Gamma, k \leftarrow 1$, and $m \leftarrow(s+t) / 2$.

Step 1.1. If $k \notin \Gamma^{\prime}$, go to Step 1.3.

Step 1.2. If $\max _{j}\left\{\beta_{j}^{k, c}\right\}>m$, let $\Gamma^{\prime} \leftarrow \Gamma^{\prime} \backslash\{k\}$.

Step 1.3. Let $k \leftarrow k+1$. If $k \leqslant n$, go to Step 1.1.

Step 1.4. If $\Gamma^{\prime}=\varnothing$, stop. There is no remaining outranking solution. Let $\rho \leftarrow\left|\Gamma^{\prime}\right|$, if $O_{1} \leqslant \rho \leqslant O_{2}$ stop, the desired number of outranking solutions has been obtained. The index set $\Gamma^{\prime}$ contains the list.

Step 1.5. If $\rho>O_{2}$, let $t \leftarrow \max \{0, t-m / 2\}$, then go to Step 1.0 .

Step 1.6. Let $s \leftarrow \min \{1, s+m / 2\}$, then go to Step 1.0 . 